\title{
Anterior uveitis in a patient receiving evolocumab
}

\author{
David Vega-Morales*, Brenda R. Vázquez-Fuentes, Jorge A. Esquivel-Valerio, and \\ Dionicio A. Galarza-Delgado
}

Rheumatology Service, Department of Internal Medicine, "Dr. José Eleuterio González" University Hospital, Universidad Autónoma de Nuevo León, Monterrey, Nuevo Leon, Mexico

\begin{abstract}
A 48-year-old male with a history of familial hypercholesterolemia treated with evolocumab subcutaneous since 2017 developed recurrent episodes of acute uveitis. The most common diseases associated with uveitis were ruled out. Physical examination revealed an arcus senilis in both eyes. To the best of our knowledge, there have been no cases of uveitis reported with the use of evolocumab. We discuss a possible mechanism for the development of uveitis in this case. We conclude that acute anterior uveitis is a possible side effect related to the use of this medication.
\end{abstract}

Key words: Evolocumab. Uveitis. Hypercholesterolemia. Corneal arcus. Hyperlipidemia.

\section{Introduction}

Anterior uveitis has a wide range of etiologies that can require specific treatment ${ }^{1,2}$. Evolocumab is a monoclonal antibody that neutralizes the proprotein convertase subtilisin/kexin type $9^{3,4}$ that leads to an increase in the number of low-density lipoprotein (LDL) receptors, ultimately improving absorption of LDL particles 5 .

We present the case of a patient with anterior uveitis that is possibly associated with evolocumab.

\section{Case description/clinical observation}

A 48-year-old male with a history of stable angina and familial hypercholesterolemia had been treated with $140 \mathrm{mg}$ of evolocumab subcutaneous QOW since February 2017, $10 \mathrm{mg}$ of rosuvastatin daily, and $135 \mathrm{mg}$ of fenofibrate daily, and was evaluated with a history of painful non-recurrent oral aphthae and mechanical back pain of 1 year of evolution. In March 2018 and April
2018, he developed a "red eye" with blurred vision. This was diagnosed as non-granulomatous unilateral acute anterior uveitis of his right eye. The patient had had a previous episode of uveitis in August 2017. The uveitis resolved without sequelae with a visual acuity of 20/20, a stable intraocular pressure of $13 \mathrm{mmHg}$, refraction for near vision exclusively, no retrokeratic deposits, and a normal fundus for both eyes.

Psoriasis, non-specific chronic ulcerative colitis, Crohn's disease, systemic lupus erythematosus (SLE), Behcet's disease, sarcoidosis, Sjögren, spondyloarthritis $(\mathrm{SpA})$, psoriatic arthritis, and reactive arthritis were ruled out by clinical history and physical examination. At the ophthalmological examination, the patient had arcus senilis in both eyes (Fig. 1).

Laboratory tests for human leukocyte antigen (HLA) B27, rheumatoid factor, anti-cyclic citrullinated peptide, antineutrophil cytoplasmic antibodies by immunofluorescence, anti-dsDNA, anti-Ro, anti-La, and anti-Sm were negative. Other results were an erythrocyte sedimentation 


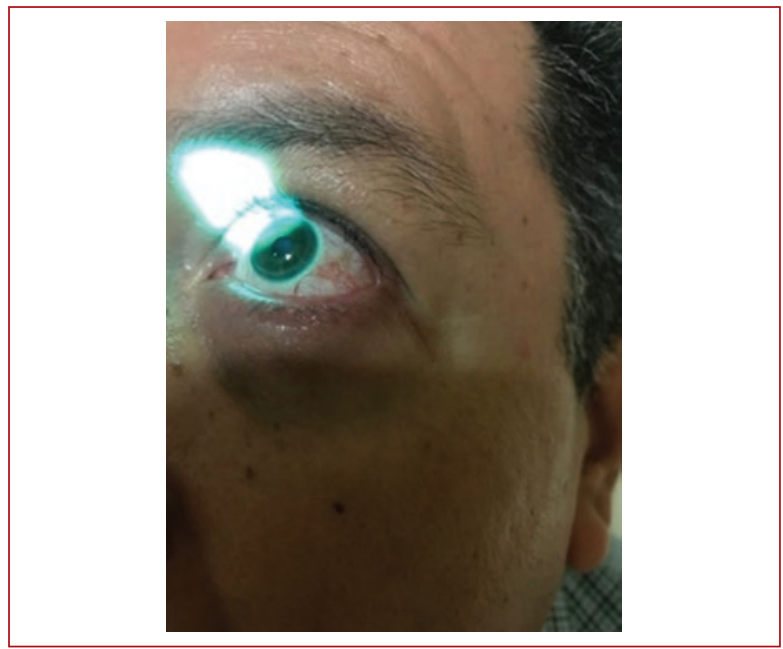

Figure 1. A corneal arch is visible when the patient gazes upward.

rate $17 \mathrm{~mm} / \mathrm{h}$, C-reactive protein $0.53 \mathrm{mg} / \mathrm{dL}$, anti-nuclear antibodies (HEp2) 1:160 by indirect immunofluorescence diffuse pattern, and anti-DFS70 $0.16 \mathrm{RU} / \mathrm{mL}$. X-rays of the lumbar spine and a magnetic resonance imaging (MRI) of the sacroiliac bones showed spondylolisthesis at L5-S1 without sacroiliitis.

Written consent from the patient and approval by the Institutional Ethics Committee following the tenets of the Declaration of Helsinki was obtained.

\section{Discussion}

The corneal arch, also known as arcus senilis or gerontoxon due to its high incidence among the elderly, is a common degeneration. It appears as a whitish ring in the stromal layer of the peripheral cornea. Its histopathology is characterized by lipid deposition (40$200 \mathrm{~nm}$ in diameter $)^{6}$ of mainly cholesterol (CHL) esters derived from LDLC, and to a lesser degree, from phospholipids and triglycerides. It is located in the extracellular matrix of the corneal stroma, under Bowman's membrane and near the perilimbal area, which is highly vascularized. A transition zone with a normal corneal appearance separates the limbic zone from the area affected by the lipid deposit (Vogt's lucid interval) ${ }^{7}$, and a relationship with hypercholesterolemia and cardiovascular disease has been demonstrated ${ }^{8}$.

The suggestion that the corneal arch is analogous to atherosclerosis was presented more than two centuries ago and seems logical because of their histological similarities. The cornea and blood vessels are composed of connective tissue in the medial zone; i.e., the corneal stroma and the media layer of the artery each contain only one type of cell, fibroblasts, and smooth muscle, respectively. The deposition of lipids in the cornea is visible and easy to study, adding additional interest to what is happening in the cornea ${ }^{9}$.

Koren et al. reported in the 12-week MENDEL study that monotherapy with evolocumab at a dose of 105 or $140 \mathrm{mg}$ every 2 weeks reduces LDL CHL by approximately $30 \%$ and increases high-density lipoprotein CHL by approximately $10 \%{ }^{10}$.

Another study reported the use of evolocumab combined with statins reducing LDL CHL levels to a median of $0.8 \mathrm{mmol} / \mathrm{L}$ (confidence interval 0.5-1.2) and significantly reducing the risk of cardiovascular events in patients with stable atherosclerotic cardiovascular disease ${ }^{11}$.

$\mathrm{SpA}$ is the most frequent systemic disease associated with anterior uveitis ${ }^{12}$. Conversely, uveitis is the most frequent extra-articular manifestation among patients with SpA. It affects approximately one-third of patients. In this population, uveitis is classically anterior $(90.5 \%)$, acute $(88.7 \%)$, unilateral $(87.3 \%)$, and in about half of patients, recurrent ${ }^{2,13}$.

Although it is one of the most frequent causes of acute anterior uveitis, and in clinical practice, it could be the first manifestation of $\mathrm{SpA} \mathrm{A}^{13,14}$, uveitis is usually associated with the HLA-B27, and the patient did not meet the criteria for a diagnosis of SpA. It has been reported that about a third of patients under 45 years of age with inflammatory lumbar pain with an evolution of more than 3 months may have spondyloarthropathy ${ }^{15}$.

Ocular disease in Behçet's disease is common and may be associated with a poor functional prognosis. Uveitis is the most common ocular manifestation of Behçet's disease and represents a diagnostic criterion. Ocular involvement at the onset of the disease is $20 \%$ and may appear 2-3 years after the onset of extraocular signs. Uveitis can be anterior, intermediate, posterior, or panuveitis. Anterior uveitis is rarely isolated and is often accompanied by posterior involvement. Anterior uveitis is always non-granulomatous and sometimes associated with hypopyon. Subsequent involvement may include the presence of hyalitis and retinal vasculitis, mainly venous and often occlusive, and macular edema and/or foci of necrotizing retinitis. Male patients with an earlier age at onset and severe tissue damage at the time of presentation have an increased risk of severe visual loss overtime ${ }^{16}$. The patient did not have genital ulcers, a positive pathergy test, or any family history or other data associated with Behçet's nor did he met the classification criteria for this disease ${ }^{17}$, 
although he did have oral aphtha that was typical of Behçet's.

The clinical manifestations of inflammatory bowel disease (IBD) are not restricted to the gastrointestinal tract. A significant number of patients have involvement in other organs and systems. The visual system is one of the most frequently affected, mainly due to inflammatory disorders, such as episcleritis, uveitis, and scleritis. A critical review of the literature regarding ocular involvement in IBD was carried out. Episcleritis, the most common ocular extra-intestinal manifestation (EIM), seems to be more associated with IBD activity compared to other ocular EIMs. In patients with IBD, anterior uveitis has an insidious onset. It is long-standing and bilateral and is not related to the activity of the intestinal disease ${ }^{18}$. The patient denied any inflammatory bowel manifestations, so this diagnosis was unlikely.

Psoriatic arthritis is an inflammatory arthropathy associated with psoriasis and should be suspected in a patient with an asymmetric pattern of joint distribution that may have additional clinical features, such as dactylitis, enthesitis or inflammatory-type back pain, and a negative rheumatoid factor. Iritis or uveitis occurs in $7 \%-18 \%$ of cases, is more frequently bilateral, but is usually found in patients with spinal involvement ${ }^{19,20}$. The patient did not have dactylitis, enthesitis, or psoriasis, although he did have a negative rheumatoid factor which did not meet the criteria for psoriatic arthritis.

Sarcoidosis is a chronic granulomatous multisystemic disease with a wide range of clinical presentations due to the immune response of the host to a persistently unknown antigen. The most frequent symptoms associated with sarcoidosis are fever, arthralgias, parotid gland enlargement, cough, dyspnea, chest pain, weakness, malaise, night sweating, fatigue, and weight loss. However, approximately half of the patients are asymptomatic. Ocular involvement occurs in $20-80 \%$ of patients at any time during the clinical course of the disease. Sarcoidosis can affect any ocular structure with anterior uveitis being the most common ocular manifestation ${ }^{21,22}$. The patient did not have African-American ancestry, no mediastinal lymph nodes, hypercalcemia, or other lesions associated with sarcoidosis.

Ocular manifestations in SLE are quite common and may be a hallmark of the disease. Furthermore, medications used in the treatment of SLE can cause eye problems such as cataracts or retinopathy ${ }^{23}$. However, SLE is a very rare cause of uveitis and the reported prevalence of SLE in patients with uveitis fluctuates between $0.1 \%$ and $5 \%{ }^{24}$. Dry eye syndrome (keratoconjunctivitis sicca) is the most common ocular feature of SLE (in about one-third of patients) and it is often associated with secondary Sjögren's syndrome. In general, the symptoms are relatively mild (irritation and redness), but there may be severe pain and visual loss. Other less frequent alterations are episcleritis, scleritis, non-infectious conjunctivitis, keratitis, and rarely, anterior uveitis; the latter more commonly associated with scleritis or posterior intraocular inflammation ${ }^{25}$. The patient had positive antinuclear antibodies (ANA), SLE-specific antibodies and clinical manifestations were absent; however, SLE is more common in women, and the lupus classification criteria were not met. The finding of a positive ANA could be explained by the use of monoclonal antibody therapy.

Some of the most common infectious causes of uveitis include herpes simplex, herpes zoster, toxoplasmosis, cytomegalovirus, syphilis, and tuberculosis. Most uveal tract infections cause characteristic changes that can be recognized with an ophthalmological evaluation. Both the rheumatologist and the ophthalmologist should be aware that before beginning immunosuppressive therapy, infectious causes must be ruled out ${ }^{26}$. In this patient, the ophthalmologist ruled out an infection.

The correlation between a corneal arch and serum lipid levels is supported by the lipid nature of the corneal deposit, a higher incidence of arcus in familial hyperlipidemia, and a variety of associated studies. Non-esterified $\mathrm{CHL}$ deposits have been detected with fluorescent dye in the limbal corneas and conjunctiva of hypercholesterolemic rabbits. Corneal arch regression has been demonstrated in hyperlipidemic animal models after the cessation of diet-induced hyperlipidemia, but this regression has never been demonstrated with lipid-lowering therapy in human subjects ${ }^{9}$.

What has been reported is that a history of cardiovascular disease, dyslipidemia, and a corneal arch in a patient under 50 years of age support a diagnosis of familial hypercholesterolemia and a higher risk for atherosclerotic coronary disease. The corneal arch reflects a generalized lipid tissue deposition and correlates with calcified atherosclerosis and xanthomatosis in patients with familial hypercholesterolemia ${ }^{27}$.

Adverse events (AEs) in the Open-Label Study of Long-term Evaluation Against LDL-C were as follows: in the standard care group, 269 patients $(73.1 \%)$ reported any $\mathrm{AE}$, and only $20(5.4 \%)$ reported back pain. Moreover, in the evolocumab group, 599 patients (81.4\%) reported any $A E$, and 48 patients (6.5\%) had back pain. The researchers considered AEs as possibly related to evolocumab in only $5.6 \%$ of all cases. Hence, the referred back pain in our patient could be secondary to 
his lumbar retrolisthesis reported in the MRI, not the evolocumab ${ }^{28}$.

Uveitis, however, to the best of our knowledge, has not been reported with the use of evolocumab. Uveitis could be idiopathic or secondary to an undifferentiated spondyloarthropathy since the patient referred to lower back pain, but did not meet ASAS criteria for spondyloarthropathy and uveitis in $\mathrm{SpA}$ is acute and explosive with significant anterior chamber (AC) inflammatory cell reaction, extensive protein exudation, a tendency for developing iris posterior synechiae, and is accompanied by red eye, significant pain, photophobia, and blurred vision, none of which were present in this patient. Nevertheless, evolocumab is a new drug and its possible long-term adverse effects are still being studied. We consider it important to report this association. A hypothetical explanation could be that the patient with a corneal arch presents a dissolution of $\mathrm{CHL}$ particles that could induce an antigenic immune response or that the release of $\mathrm{CHL}$ produces an inflammatory reaction causing uveitis, similar to the mechanism of $\mathrm{CHL}$ crystals, in which hypercholesterolemia leads to the accumulation of $\mathrm{CHL}$ in macrophages and other immune cells that promote an inflammatory response, including increased Toll-like receptor (TLR) signaling, inflammasome activation, and the production of monocytes and neutrophils in the bone marrow and spleen, and at the cellular level. There is only one major problem with this hypothetical pathogenic mechanism. The cornea and AC of the eye are known to be immune-privileged sites. The avascularity of the cornea, the scant existence of APCs, mostly Langerhans cells and interdigitating cells, and the posterior boundary with the AC make the cornea a site of immune privilege. This is why corneal transplants have the highest survival rate of all tissue-organ transplants in the human body. On the other hand, the $\mathrm{AC}$ experiences the $\mathrm{AC}$-associated immune deviation phenomenon, which is characterized by no or poor immune response to antigen presentation through this compartment. Large molecular weight proteins, like ova-albumin, presented through the AC can abolish a delayed-type hypersensitivity (DTH) response to the same proteins in the skin after a later Ag challenge. Hence, if some CHL, partly dissolved or molecularly modified by evolocumab, are presented as autoantigens through the cornea or the $\mathrm{AC}$, the most probable immune response would be none. However, the immune privilege of these ocular structures may be easily lost if corneal neovascularization or previous intraocular inflammation has occurred in the eye. Activation of
TLR signaling leads to a decrease in CHL efflux, which results in a greater accumulation of $\mathrm{CHL}$ and the amplification of inflammatory responses ${ }^{29}$.

We see it as an analogy to patients who have a crystal arthropathy with hypouricemic drug treatment. It is now well-known that evolocumab is one of the most powerful hypocholesterolemic drugs available today, which could cause this $\mathrm{AE}$ during the dissolution of $\mathrm{CHL}$ crystals in the AC of the eye. This is a less frequent mechanism since the most common mechanisms of anterior uveitis are autoimmune or infectious in origin.

\section{Conclusions}

Although there are more common causes of acute anterior uveitis, because of the use of this new biological drug, of which not enough is known regarding autoimmune events, we decided to present this case, proposing that it could be related to the use of this medication.

\section{Conflicts of interest}

The authors declare that they have no conflicts of interest.

\section{Ethical disclosures}

Protection of human and animal subjects. The authors declare that no experiments were performed on humans or animals for this study.

Confidentiality of data. The authors declare that they have followed the protocols of their work center on the publication of patient data.

Right to privacy and informed consent. The authors have obtained the written informed consent of the patients or subjects mentioned in the article. The corresponding author is in possession of this document.

\section{References}

1. Calvo-Hernandez LM, Bautista-Salinas RM, Suarez-Cabrera M. Uveitis: a challenge for internist. An Med Interna. 2008;25:141-8.

2. Wach J, Maucort-Boulch D, Kodjikian L, Iwaz J, Broussolle C, Seve P. Acute anterior uveitis and undiagnosed spondyloarthritis: usefulness of Berlin criteria. Graefes Arch Clin Exp Ophthalmol. 2015;253:115-20.

3. Sabatine MS, Leiter LA, Wiviott SD, Giugliano RP, Deedwania P, De Ferrari GM, et al. Cardiovascular safety and efficacy of the PCSK9 inhibitor evolocumab in patients with and without diabetes and the effect of evolocumab on glycaemia and risk of new-onset diabetes: a prespecified analysis of the FOURIER randomised controlled trial. Lancet Diabetes Endocrinol. 2017:5:941-50.

4. Toth PP, Worthy G, Gandra SR, Sattar N, Bray S, Cheng L, et al. Systematic review and network meta-analysis on the efficacy of evolocumab and other therapies for the management of lipid levels in hyperlipidemia. J Am Heart Assoc. 2017;6:e005367. 
5. Reiss AB, Shah N, Muhieddine D, Zhen J, Yudkevich J, Kasselman LJ, et al. PCSK9 in cholesterol metabolism: from bench to bedside. Clin Sci (Lond). 2018;132:1135-53.

6. Francois J, Feher J. Arcus senilis. Doc Ophthalmol 1973;34:165-82.

7. Fernandez A, Sorokin A, Thompson PD. Corneal arcus as coronary artery disease risk factor. Atherosclerosis. 2007;193:235-40.

8. Hashemi H, Khabazkhoob M, Emamian MH, Shariati M, Fotouhi A. A population-based study of corneal arcus and its risk factors in Iran. Ophthalmic Epidemiol. 2014;21:339-44.

9. Moosavi M, Sareshtedar A, Zarei-Ghanavati S, Zarei-Ghanavati M, Ramezanfar N. Risk factors for senile corneal arcus in patients with acute myocardial infarction. J Ophthalmic Vis Res. 2010;5:228-31.

10. Koren MJ, Scott R, Kim JB, Knusel B, Liu T, Lei L, et al. Efficacy, safety, and tolerability of a monoclonal antibody to proprotein convertase subtilisin/kexin type 9 as monotherapy in patients with hypercholesterolaemia (MENDEL): a randomised, double-blind, placebo-controlled, phase 2 study. Lancet. 2012;380:1995-2006.

11. Giugliano RP, Pedersen TR, Park JG, De Ferrari GM, Gaciong ZA, Ceska $R$, et al. Clinical efficacy and safety of achieving very low LDL-cholesterol concentrations with the PCSK9 inhibitor evolocumab: a prespecified secondary analysis of the FOURIER trial. Lancet. 2017:390:1962-71.

12. Murray PI, Rauz S. The eye and inflammatory rheumatic diseases: the eye and rheumatoid arthritis, ankylosing spondylitis, psoriatic arthritis. Best Pract Res Clin Rheumatol. 2016;30:802-25.

13. Rosenbaum JT. New developments in uveitis associated with HLA B27. Curr Opin Rheumatol. 2017;29:298-303.

14. Yen JC, Hsu CA, Hsiao SH, Hsu MH. Acute anterior uveitis as a risk factor of ankylosing spondylitis a national population-based study. Int $J$ Environ Res Public Health. 2017;14:107.

15. Rasool T, Umer TP, Nazir L, Arain SR, Phulpoto K, Afzal W. Axial spond yloarthritis in patients with chronic backache using assessment of spondyloarthritis international society criteria for axial spondyloarthritis. J Ayub Med Coll Abbottabad. 2018;30:253-7.

16. Desbois AC, Terrada C, Cacoub P, Bodaghi B, Saadoun D. Ocular manifestations in Behcet's disease. Rev Med Interne. 2018;39:738-45.
17. Blake T, Pickup L, Carruthers D, Damato EM, Denniston A, Hamburger J, et al. Birmingham Behcet's service: classification of disease and application of the 2014 international criteria for Behcet's disease (ICBD) to a UK cohort. BMC Musculoskelet Disord. 2017;18:101.

18. Troncoso LL, Biancardi AL, de Moraes HV Jr., Zaltman C. Ophthalmic manifestations in patients with inflammatory bowel disease: a review. World J Gastroenterol. 2017;23:5836-48.

19. Ritchlin CT, Colbert RA, Gladman DD. Psoriatic arthritis. N Engl J Med. 2017;376:957-70.

20. Abbouda A, Abicca I, Fabiani C, Scappatura N, Peña-García P, Scrivo R et al. Psoriasis and psoriatic arthritis-related uveitis: different ophthalmological manifestations and ocular inflammation features. Semin Ophthalmol. 2017;32:715-20.

21. Cornblath WT, Elner V, Rolfe M. Extraocular muscle involvement in sarcoidosis. Ophthalmology. 1993;100:501-5.

22. Garweg JG. Sarcoidosis and uveitis: an update. Ophthalmologe 2017; $114: 525-33$

23. Sivaraj RR, Durrani OM, Denniston AK, Murray PI, Gordon C. Ocular manifestations of systemic lupus erythematosus. Rheumatology (Oxford). 2007; 46:1757-62.

24. Gallagher K, Viswanathan A, Okhravi N. Association of systemic lupus erythematosus with uveitis. JAMA Ophthalmol. 2015;133:1190-3.

25. Generali E, Cantarini L, Selmi C. Ocular involvement in systemic autoimmune diseases. Clin Rev Allergy Immunol. 2015;49:263-70.

26. Rosenbaum JT, Dick AD. The eyes have it: a rheumatologist's view of uveitis. Arthritis Rheumatol. 2018;70:1533-43.

27. Ford TJ, Rocchiccioli P. A keen eye for risk. BMJ. 2018;360:j5884.

28. Koren MJ, Giugliano RP, Raal FJ, Sullivan D, Bolognese M, Langsl G, et al. Efficacy and safety of longer-term administration of evolocumab (AMG 145) in patients with hypercholesterolemia: 52-week results from the open-label study of long-term evaluation against LDL-C (OSLER) randomized trial. Circulation. 2014;129:234-43.

29. Tall $A R$, Yvan-Charvet $L$. Cholesterol, inflammation and innate immunity. Nat Rev Immunol. 2015;15:104-16. 